\title{
A Resource Allocation Scheme for TH-UWB Networks with Multiple Sinks
}

\author{
Hwee-Xian Tan*, Mun-Choon Chan*, Peng-Yong Kong ${ }^{\dagger}$ and Chen-Khong Tham ${ }^{\dagger}$ \\ * School of Computing, National University of Singapore (NUS) \\ ${ }^{\dagger}$ Institute for Infocomm Research $\left(\mathrm{I}^{2} \mathrm{R}\right)$, Singapore \\ $\{$ hweexian, chanmc $\} @$ comp.nus.edu.sg, \{kongpy, cktham $\} @ i 2$ r.a-star.edu.sg
}

\begin{abstract}
In this work, we study the time-slot allocation problem in a multi-sink single-hop TH-UWB network scenario, where the traffic from a sensor node is anycasted via a single hop to any one of multiple sinks. The slot allocation problem is formulated as an optimization problem and shown to be NP-hard. We then present a heuristic to increase network throughput and fairness as compared to a random allocation. In the proposed heuristic, nodes that are of similar distances to any sinks are grouped together to utilize the same set of TH slots for transmissions. Simulations show that the proposed heuristic improves both throughput and fairness, scales with multiple sinks and can be used as a simple admission control mechanism.
\end{abstract}

\section{INTRODUCTION}

The adoption of Ultra Wideband (UWB) as an alternative physical (PHY) layer technology in Low-Rate Wireless Personal Area Networks (LR-WPANs) [1] has opened doors to a wider range of potentials in wireless communications [2]. According to the First Report and Order released by FCC in Feb 2002 [3], a UWB device is defined to be one whereby the fractional bandwidth is at least $20 \%$ or occupies at least 500 $\mathrm{MHz}$ of the spectrum. With UWB, nodes can achieve very high data rates using very low power consumptions. The extremely narrow pulses used in UWB transmissions also enables highprecision localization, which can be exploited for many uses such as energy conservation, provision of QoS (Quality of Service), interference management, etc.

Due to the inherent low-power and short-range characteristics of the UWB technology, it is suitable for use in sensor networks, which can be deployed for a myriad of applications ranging from periodic monitoring, industrial automation to home surveillance. The nodes in a sensor network typically sense physical data from the environment (such as temperature), and transmit the collected data to one or more centralized sinks (destinations) for processing and decision-making.

In this paper, we study a multi-sink single-hop scenario where the traffic from a sensor node is anycasted via a single hop to any one sink out of multiple sink choices. We have chosen an anycast paradigm as communication in wireless networks is inherently broadcast in nature, and cooperation between sinks should be exploited to improve the overall network performance. Unlike previous work on multiple sinks or base stations (BS) selection [4][5][6][7], we consider a THUWB (Time-Hopping) physical layer. In TH-UWB, a node uses one out of a set of pre-allocated $\mathrm{TH}$ slots to transmit each of its data packets, thus providing a form of multiple access scheme. Our work involves the allocation of a subset of TH slots to be used by a sensor for data transmissions, to achieve various network objectives such as high throughput and fairness.

We first formulate the slot allocation problem as an optimization problem and prove that it is NP-hard. We then develop heuristics for the main objectives of achieving high network throughput and fairness, by allowing nodes that are of similar distances to any sinks to compete together by using the same TH slots for transmissions. We show the efficacy of our proposed heuristics through extensive simulations.

The rest of this paper is organized as follows: Section II describes background and related work in the slotted-Aloha MAC protocol with TH-UWB and multiple sink/BS selection. Some preliminaries are presented in Section III. The proposed heuristics and corresponding performance analysis are detailed in Sections IV and V respectively. We conclude the paper in Section VI with some directions for future work.

\section{BACKGROUND AND RELATED WORK}

\section{A. Slotted-Aloha MAC Protocol}

The performance of slotted-Aloha in a TH-UWB network has been studied in [8]. In TH-UWB, each signal is transmitted over several symbols, each of which is constituted by a burst of very short pulses. As such, traditional MAC protocols that make use of carrier sensing are unsuitable for use in such impulse-based networks. In addition, the IEEE 802.15.4a standard [1] also supports the use of Aloha as the standard MAC protocol in UWB-based LR-WPANs networks.

We consider a slight variant of the slotted-Aloha MAC protocol which incorporates TH-UWB into its frame structure. In the simplified frame structure (Figure 1), there are a total of $p$ TH slots; each data packet is assumed to be transmitted within $T_{\text {slot }}$. A beacon $B$ is inserted at the start of the MAC frame for purposes such as time synchronization and localization. In each MAC frame, a sensor node may transmit a packet in only one out of $p$ possible TH slots.

\section{B. Multiple Sink Selection}

There exists some work on the multiple sink selection problem in wireless sensor networks in the literature. In the Sink-based Anycast Routing Protocol (SARP) [9], each packet is forwarded to the nearest sink instead of a fixed destination. The authors of SARP assert that the delivery of packets to 


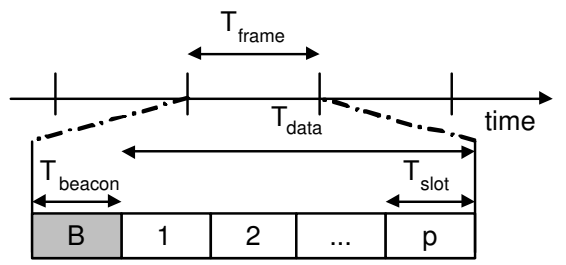

Fig. 1. Frame Structure of Slotted-Aloha with TH-UWB

the nearest sink incurs minimal bandwidth, power and delay, making SARP suitable for resource allocation applications.

Hou et al [5] study the optimal sink selection problem in which each sensor has to find an optimal route to transmit all its data to only one of the multiple sinks while maximizing the network lifetime. The authors then propose ABS (Anycast Base Station Selection) on the basis that the optimal sink for an arbitrary node is highly correlated to the sink that receives the largest volume of traffic when there is no constraint on the number of destination sinks for the node.

Unlike existing work on multiple sinks or base stations (BS) selection, we consider a TH-UWB physical layer in the network. Using TH-UWB, a node may use different timehopping slots for communication. If all the nodes are allowed to use all the TH slots for transmissions, there is high multiuser interference and low channel utilization, which reduces the overall throughput of the network. The near-far effect under such circumstances also gives rise to unfairness in the network. However, strict partitioning of $\mathrm{TH}$ codes may not yield optimal network performance, due to the inability of resource multiplexing, especially in scenarios with uneven traffic patterns. Hence, our work focuses on the allocation of $\mathrm{TH}$ slots to nodes to improve throughput while ensuring some form of fairness in the network.

\section{PRELIMINARIES}

\section{A. Network Model}

The network is modeled as a graph $G=(V, E)$ where $V=V_{s} \cup V_{r}$ and $V_{s} \cap V_{r}=\emptyset$. We let $V_{s}=\left\{s_{1}, s_{2}, \ldots, s_{n}\right\}$ represent the set of sensor (source) nodes in the network and $V_{r}=\left\{r_{1}, r_{2}, \ldots, r_{m}\right\}$ represent the set of sinks (destinations) in the network. The total number of nodes and sinks in the network are denoted as $n=\left|V_{s}\right|$ and $m=\left|V_{r}\right|$ respectively. The edge $e_{i j} \in E$ represents the existence of a wireless link between an arbitrary sensor node $s_{i}$ and an arbitrary sink $r_{j}$. We assert that each sensor is connected to at least one sink via a single hop. The node degree and supported bandwidth of sink $r_{i}$ are denoted as $d_{v}(i)$ and $w_{i}$ respectively.

\section{B. Physical Model}

The set of TH slots available in a TH-UWB network is represented by $C=\left\{c_{1}, c_{2}, \ldots, c_{p}\right\}$, where $p=|C|$ is the total number of TH slots. Each sensor $s_{i}$ is allocated a set of $\mathrm{TH}$ slots $h_{i} \subseteq C$ which it uses to transmit its packets to the sinks. If $\left|h_{i}\right| \geq 1, s_{i}$ will uniform-randomly select a $\mathrm{TH}$ slot from $h_{i}$ to transmit each of its packets.

\section{Traffic Model}

We consider a Poisson traffic model, whereby a data packet of length $L_{p}$ is generated at a rate of $\lambda$ packets per second in each sensor. Due to the broadcast nature of wireless communications, the packet transmission from an arbitrary sensor $s_{i}$ may reach more than one sink. Hence, the flow demand $f_{i}$ from $s_{i}$ can be modeled as:

$$
f_{i}=\sum_{j=1}^{m} f_{i j} \cdot s_{i j}=\lambda \cdot L_{p} \quad r_{j} \in V_{r}
$$

where $f_{i j}$ denotes the flow from $s_{i}$ to $r_{j} ; s_{i j} \in\{0,1\}$; and $s_{i j}=1$ if $e_{i j} \in E$ and $s_{i j}=0$ otherwise.

\section{Throughput Model}

The throughput achieved by a sensor $s_{i}$ at a sink $r_{j}$ is dependent on its probability of successful transmissions at $r_{j}$, which is defined according to the model presented in [10]:

$$
P_{s(i, j)}=e^{-\frac{R_{t} N_{0} P L(i)}{P_{0}}} \prod_{k=1}^{q} 1-\frac{R_{t} \frac{1}{\left|h_{i}\right|}}{R_{t}+\frac{P L(k)}{P L(i)}}
$$

where $R_{t}$ is the SINR threshold; $N_{0}$ is the noise power; $P_{0}$ is the transmit power; and $q$ is the number of interferers. $P L(i)$ is the UWB pathloss of sensor $s_{i}$ at distance $d_{i j}$ from sink $r_{j}$, and is defined as:

$P L(i)= \begin{cases}d_{i j}{ }^{2} & \text { if } d_{i j} \leq d_{0}, d_{0}=1 m \\ P L\left(d_{0}\right)+d_{i j}{ }^{\beta}+\left(\frac{f}{f_{c}}\right)^{2} & \text { otherwise }\end{cases}$

where $\beta=1.79$ is the pathloss exponent in a UWB residential line-of-sight (LOS) environment; $f$ is the channel frequency; and $f_{c}$ is the center frequency.

\section{E. Problem Formulation}

The main focus of our work is to find a mapping $M$ which allocates a set of $\mathrm{TH}$ slots to each sensor $s_{i}$ in order to optimize a particular network objective $Q$. Hence, $M=\left\{M_{1}, M_{2}, \ldots, M_{n}\right\}$, where $M_{i}=\left(h_{i}, s_{i}\right)$; and $Q$ may be any pre-specified QoS requirement such as throughput, fairness or energy consumption.

We assume that the network objective $Q$ is to maximize the total throughput, and refer to this problem as $M A X$ THROUGHPUT:

$$
P_{\text {total }}=\sum_{i=1}^{n} \sum_{j=1}^{m} P_{s(i, j)}
$$

Theorem 1: Finding a mapping $M$ which assigns a set of TH slots $h_{i}$ to each node $s_{i}$ while maximizing $P_{\text {total }}$ is NPhard.

Proof 1: We show that MAX-THROUGHPUT is NP-hard by reducing the well-known NP-hard problem Generalized Assignment Problem (GAP) to it. In GAP, there is a set $S$ of $n$ items and a set $B$ of $m$ bins; each bin $b_{j} \in B$ is associated with a budget $w_{j}$. For each bin $b_{j}$, each item $s_{i} \in S$ is associated with a profit $p_{i j}$ and a weight $w_{i j}$. The objective of GAP is to find a subset $U \subseteq S$ and an assignment from $U$ to the 
bins, i.e., $f: U \rightarrow B$, such that: (i) the sum of the weights of the assigned items in each bin $b_{j}$ does not exceed $w_{j}$; and (ii) the maximum profit from all possible assignments is obtained. Formally, GAP can be formulated as follows:

$$
\max \sum_{i=1}^{n} \sum_{j=1}^{m} p_{i j} \cdot s_{i j}
$$

where $s_{i j} \in\{0,1\}$; and $s_{i j}=1$ if item $s_{i}$ is placed in bin $b_{j}$ and $s_{i j}=0$ otherwise.

We map $S$ to $V_{s}$ and $B$ to $V_{r}$, such that the budget $w_{j}$ that is associated with each bin $b_{j}$ is equivalent to the maximum bandwidth $w_{j}$ that is supported by sink $r_{j}$. The profit $p_{i j}$ and weight $w_{i j}$ that is contributed by item $i$ when it is placed in bin $j$ is equivalent to the throughput $P_{s(i, j)}$ and interference $I_{i j}$ respectively, of a sensor $s_{i}$ at sink $r_{j}$. Here, we have omitted the complexity of computing $P_{s(i, j)}$ and $I_{i j}$, which are dependent on the set of TH slots being assigned to $s_{i}$. This implies that a solution for MAX-THROUGHPUT, which is maximizing $P_{\text {total }}$, will be a solution for GAP; therefore MAX-THROUGHPUT is at least NP-hard.

Theorem 2: The algorithmic complexity of $M A X$ THROUGHPUT is $\Theta\left(2^{n p}\right)$.

Proof 2: There are a total of $p \mathrm{TH}$ slots and $n$ sensors in the network. Each sensor may be allocated any number of TH slots for transmissions; hence, there are $2^{p}-1$ ways of selecting $h_{i} \subseteq C$ for each sensor. Since there are $n$ sensors, the total complexity of MAX-THROUGHPUT is $\Theta\left(2^{n p}\right)$.

The throughput optimization problem is formulated as:

Max: Ptotal

subject to:

$$
\begin{gathered}
c_{i} \in \bigcup_{j=1} h_{j} \quad \forall c_{i} \in C ; \forall h_{j} \subseteq C \\
\sum_{j=1}^{m} P_{s(i, j)}>0 \quad s_{i} \in V_{s} ; r_{j} \in V_{r} \\
\sum_{i=1}^{d_{v}(j)} f_{i j} \cdot s_{i j} \leq w_{j} \quad \forall s_{i} \in V_{s}, r_{j} \in V_{r} \\
\sum_{j=1}^{m} s_{i j} \geq 1 \quad \forall s_{i} \in V_{s}, r_{j} \in V_{r} \\
s_{i j} \in\{0,1\}
\end{gathered}
$$

Equation 6 ensures that all the available codes $r_{i} \in R$ must be allocated to at least one sensor for data transmission, which maximizes the utilization of all available resources. Equation 7 ensures that no sensor in the network will be starved. Equation 8 ensures that the sum of all flows to any particular sink will not exceed its maximum bandwidth. Equations 9 and 10 ensure that each sensor must be allocated to at least one sink.

\section{HEURISTICS}

The presence of multiple sinks in the network calls for a resource allocation scheme that can maximize the overall network utilization and efficiency. In wireless networks with multiple sinks, many packets are duplicated at the sinks due to the broadcast nature of wireless communications. Although this improves the reliability of the network by creating redundancy at multiple sinks, the advantage of having multiple sinks in the network is not fully exploited. In this section, we describe a greedy heuristic that: (i) effectively leverages on the presence of multiple sinks to improve throughput; (ii) achieves some form of fairness; and (iii) can be used as an admission control mechanism to provide QoS.

When there are multiple sensor nodes transmitting at the same time in the shared communication channel, typically only the signal with the highest SINR value above a particular receiver threshold will be accepted at the receiver (or sink). This results in under-utilization of the available network resources when the same packet is received by more than one sinks. Hence, the main objective of our proposed heuristic is to reduce duplicates and increase the number of successful simultaneous transmission by different nodes, while maintaining some form of fairness in the network.

In the presence of multiple sinks, the overall probability of successful transmission of sensor $s_{i}$ can be modeled as:

$$
P_{s(i)}=1-\prod_{j=1}^{m} 1-P_{s(i, j)}
$$

where $P_{s(i, j)}$ is the probability of successful transmission between sensor $s_{i}$ and sink $r_{j}$ as defined in Equation 2.

We assume that the algorithm is run by a central controller. First, the distances among the sensors and sinks are collected. The shortest distance of sensor $s_{i}$ to any sink is defined as:

$$
d(i)^{\prime}=\min \left(d_{i 1}, d_{i 2}, \ldots, d_{i m}\right)
$$

where $d_{i j}$ is the distance between $s_{i}$ and a particular sink $r_{j}$.

The algorithm maintains three lists: (1) $L_{1}$ : list of sensors with unallocated time slots; (2) $L_{2}$ : list of sensors whose time slot allocations are currently being determined; and (3) $L_{3}$ : list of sensors with allocated time slots. In the beginning, all the sensors are in list $L_{1}$ and sorted in ascending order according to their respective values of $d(i)^{\prime}$. Sensors are always removed from $L_{1}$ in this order. $L_{2}$ and $L_{3}$ are initially empty.

The algorithm proceeds in rounds of considering one sensor at a time until $L_{1}$ is empty and $L_{3}$ contains all the sensors. In each round, the algorithm picks sensor $s_{t}$ with the minimum $d(i)^{\prime}$ from $L_{1}$ and attempts to insert it into $L_{2}$. The values of $P_{s(i, j)}$ of each sensor that is currently in $L_{2}$ is computed to take into account the interference caused by all elements in $L_{2}$ as well as $s_{t}$. The value of $\left|h_{i}\right|$ is also updated such that $\left|h_{i}\right|=\frac{p \cdot\left(\left|L_{2}\right|+1\right)}{n}$, where $\left|L_{2}\right|$ is the size of list $L_{2}$.

We compare the probability of successful transmission of each sensor $P_{s(i)}, s_{i} \in L_{2}$ with its corresponding probability of successful transmission when random allocation is used, i.e. $P_{r(i)}$. The random allocation refers to the case whereby 


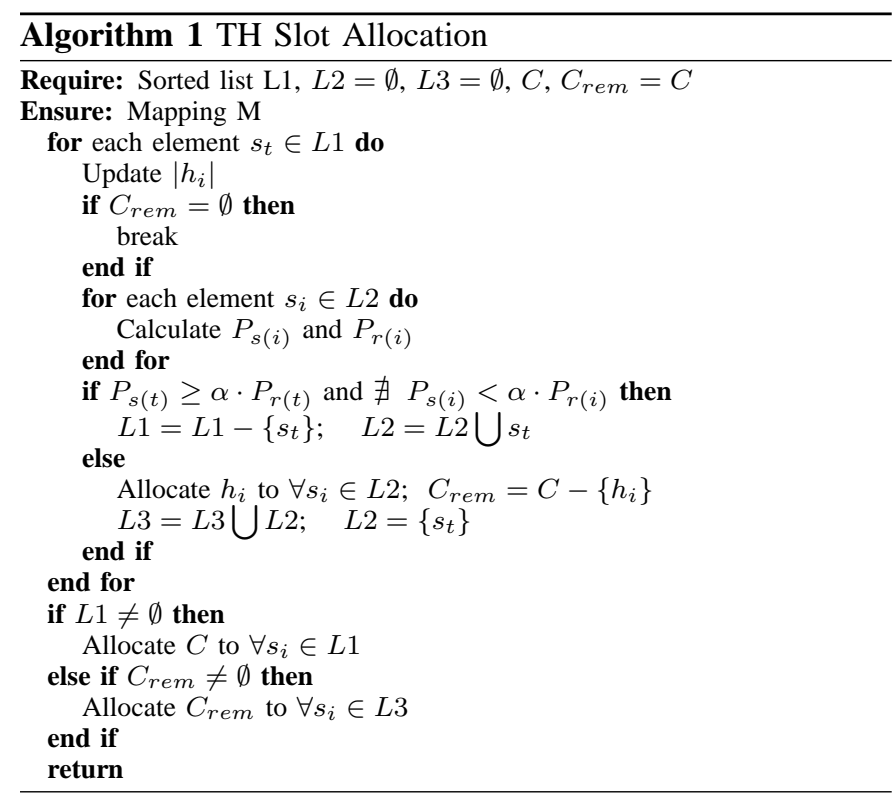

all the sensors uniform-randomly select a slot from the entire set $C$ of available TH slots for each data transmission. The value of $P_{r(i)}$ is calculated according to Equations 2 and 11 with $q=n-1$ and $\left|h_{i}\right|=p$. The sensor $s_{t}$ is removed from $L_{1}$ and inserted into $L_{2}$ iff $P_{s(t)} \geq \alpha \cdot P_{r(t)}$ and:

$$
\nexists P_{s(i)}<\alpha \cdot P_{r(i)} \quad \forall s_{i} \in L_{2}
$$

where $\alpha$ is a tunable parameter usually between 0 and 1 . $\alpha$ controls the amount of tolerable interference that is acceptable above the random allocation. It is needed to account for the fact that Equations 2 and 11 assume that the data queues are always backlogged. Note that when $\alpha=0$, the scheme becomes a random allocation. For light to medium loads, $\alpha=1$ may be too conservative and a smaller $\alpha$ may be needed. The impact of $\alpha$ is investigated through simulations in the section on performance evaluation.

If Equation 13 is not satisfied, $s_{t}$ is not added to $L_{2}$. Instead, the current sensors in $L_{2}$ are allocated the next $\left|h_{i}\right|=\frac{p \cdot\left|L_{2}\right|}{n}$ TH slots that have not been assigned to any sensor, and moved from $L_{2}$ to $L_{3}$. The algorithm proceeds with inserting $s_{t}$ to (the now empty) $L_{2}$ and the cycle repeats with the next sensor in $L_{1}$. The algorithm terminates when all the sensors have been allocated $\mathrm{TH}$ slots or when there is insufficient slots to be allocated to the sensors. In the former case, the excess time slots, if any, are allocated randomly among the sensors. In the latter case, the remaining sensors in $L_{1}$ and $L_{2}$ pick from the entire set of $p$ TH slots for transmissions.

The algorithm achieves the following: By grouping together nodes that are of similar distances to the sinks, the number of successful simultaneous transmissions by different sensors can be increased. Such grouping also has the benefit of achieving better fairness. Next, by using random allocation as the baseline, we ensure that there will be improvement over random allocation whenever possible. The pseudo code of the algorithm is presented in Algorithm 1.
TABLE I

SimUlation PARAMETERS

\begin{tabular}{|c||c|}
\hline \multicolumn{1}{|c|}{ Parameter } & Value \\
\hline Terrain size & $8 \mathrm{~m} \times 8 \mathrm{~m}$ \\
\hline Number of sensors $n$ & 16 to 256 \\
\hline Number of sinks $m$ & $\{1,2,3,4,5\}$ \\
\hline Transmission power $P$ & $-14.32 \mathrm{dBm}$ \\
\hline Channel frequency $f$ & $4 \mathrm{GHz}$ \\
\hline Center frequency $f_{c}$ & $4492.8 \mathrm{MHz}$ \\
\hline Channel bandwidth $B$ & $499.2 \mathrm{MHz}$ \\
\hline Number of TH slots $p$ & 8 \\
\hline Slot length $T_{\text {slot }}$ & $0.2304 \mathrm{~ms}$ \\
\hline Frame length $T_{\text {frame }}$ & $\approx 2 \mathrm{~ms}$ \\
\hline Packet arrival rate (per source) $\lambda$ & 100 to 1000 \\
\hline Packet length $L$ & 56 bytes \\
\hline
\end{tabular}

\section{Performance Evaluation}

We study the performance of our proposed heuristic using Qualnet [11], a scalable network simulator. The PHY and MAC layers in Qualnet are modified in order to model the characteristics of a slotted-Aloha MAC protocol in a THUWB physical medium. We do not consider the effects of time synchronization and localization in the slotted-Aloha MAC protocol. Due to the presence of multiple sinks in the network, each packet may be received by more than one sink. We assume that the central controller, which executes the algorithm, is connected through a wired network to the data sinks; hence, only the sink that first receives the packet sends an implicit ACK to the sensor. In the event of packet losses arising from the imperfect physical channel, interference or packet collisions, each packet is allowed up to 3 retransmissions, after which the packet is discarded. We make use of the Poisson traffic model with a mean packet rate $\lambda$. Each simulation setup is run for 10 different seeds to minimize any arbitrary randomness. Table I summarizes the simulation parameters. Based on the parameters used, the maximum transmission range without interference is about $8 \mathrm{~m}$.

\section{A. Network with Varying Traffic Loads}

Figure 2 shows the performance of our proposed heuristic in a network with 16 sensor sources and 2 sinks The traffic load is varied by increasing $\lambda$ from 100 packets/second to 1000 packets/second. As the traffic load increases, the network becomes increasingly congested, resulting in excessive collisions and low Packet Delivery Ratios (PDRs), as shown in Figure 2(a). Despite this, the proposed heuristic can still achieve higher normalized throughputs and lower delays, as shown in Figures 2(b) and 2(c) respectively. The efficiency of the different allocation schemes is illustrated in Figure 2(d), in which the number of retransmissions required per data packet in the proposed scheme is always lower than that required in the random allocation scheme. The efficiency of the MAC protocol has a considerable impact on energy consumption and network lifetime. 


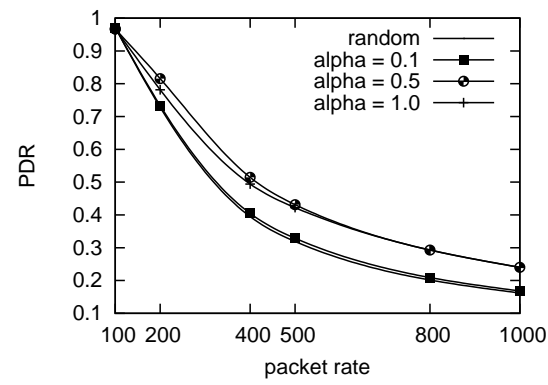

(a) PDR

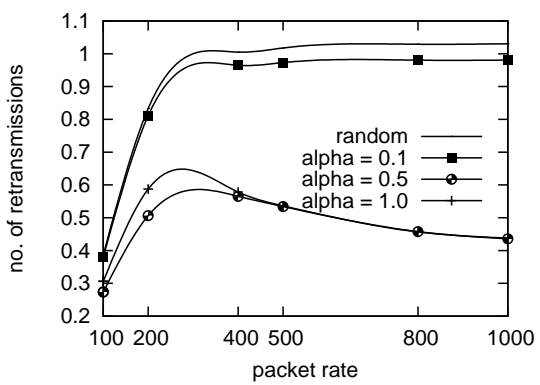

(d) Efficiency

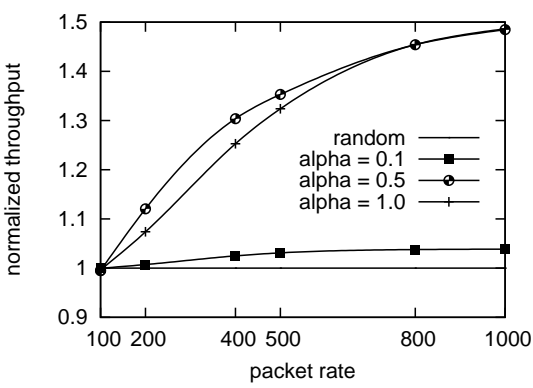

(b) Normalized Throughput

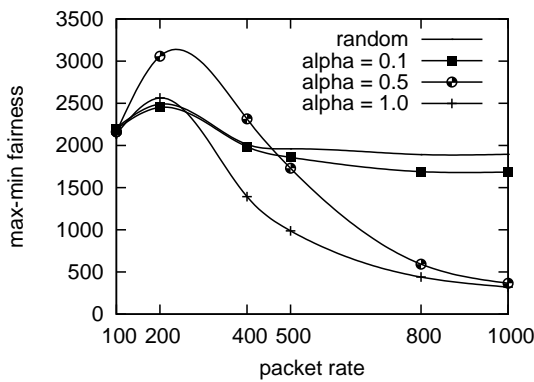

(e) Max-Min Fairness

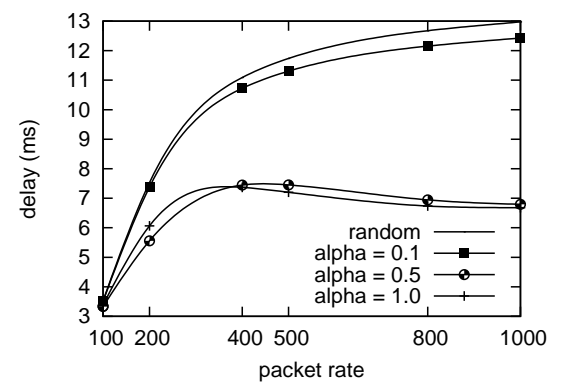

(c) Delay

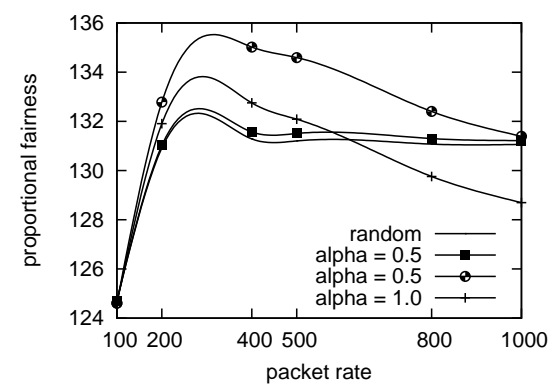

(f) Proportional Fairness

Fig. 2. Performance of proposed heuristic in network with 16 sensor sources and 2 sinks.

It can be observed that with a very small $\alpha(0.1)$, the performance is close to that of random allocation. In this scenario, $\alpha=0.5$ performs slightly better than a value of 1.0 as the interference estimation using $\alpha=1.0$ is too conservative.

We also study the fairness of the different allocation schemes using two different metrics, viz. max-min fairness and proportional fairness. Max-min fairness maximizes the minimum throughput (or packets received) by any node in the network, often at the cost of network throughput, while proportional fairness attempts to strike a balance between fairness and network utilization. In Figure 2(e), the proposed scheme with $\alpha=0.5$ is able to achieve higher max-min fairness for $\lambda \leq 400$ where the PDR $\geq 0.5$. When the traffic load is high, the random allocation can achieve better max-min fairness as sensors that are further away have less available resources (time slots) in the proposed scheme, and excessive interferences cause these sensors to achieve much lower throughput than those that are located nearer to the sinks. However, this phenomenon occurs only when the network is operating at extremely high loads with PDRs of less than 0.5. In Figure 2(f), our proposed scheme is able to achieve better proportional fairness as this metric aims to achieve fairness at low cost to network utilization.

We have studied the performance of our scheme for larger network sizes ranging from 32 sensors to 256 sensors, with 2 sinks for data collection, and obtained similar results.

\section{B. Network with Varying Number of Sinks}

We study the performance of the network with varying number of sinks and a proportional increase in the number of sensor sources in the network. The number of sinks are varied from 1 to 5 , and the corresponding number of sensors are varied from 8 to 40 in increasing step size of 8 . The traffic arrival rate $\lambda$ for each sensor is fixed at 400 packets per second.

It can be seen from Figures 3(a) and 3(b) that our proposed heuristic can achieve throughput improvements of up to $30 \%$ with 2 sinks and 16 sensors, while incurring lower delays than the random allocation scheme. As the number of sinks (and sensors) in the network increase, the throughput improvement decreases, but is still able to achieve at least $10 \%$ improvement. In general, the amount of improvement achievable depends heavily on the sensor and sink placements and does not increase with the number of sinks if the sinks are placed too close together. Varying levels of fairness improvements are also attained using different $\alpha$ values, as shown in Figure 3(c).

\section{Network with Admission Control}

We study the performance of the proposed heuristic as an admission control mechanism, whereby sensors that have insufficient codes for allocation (as described in Section IV) will not be admitted into the system. We implement the random allocation scheme in the same manner. The desired QoS metric is defined to be the probability of successful transmissions $P_{s}$ and is set to vary from 0.1 to 1 .

Table II shows the performance of the proposed heuristic with 32 sensor sources, 2 sink nodes and $\lambda=200$. Here, $N_{a}$ refers to the average number of sensor nodes that are admitted into the system and $N_{s}$ refers to the fraction of admitted sensors that are able to satisfy the required level of QoS.

At small values of required $P_{s}$, the proposed scheme (with $\alpha=1$ ) tends to be overly conservative and over-allocate the 


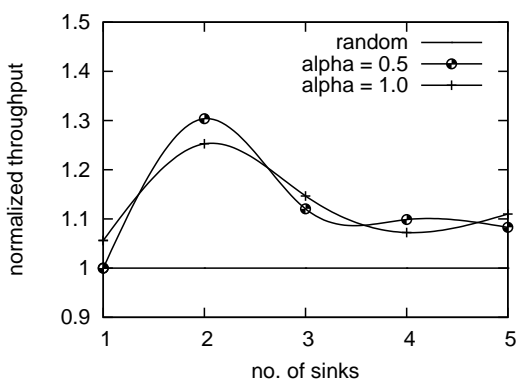

(a) Normalized Throughput

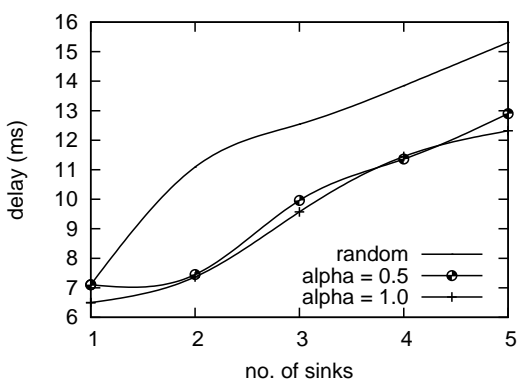

(b) Delay

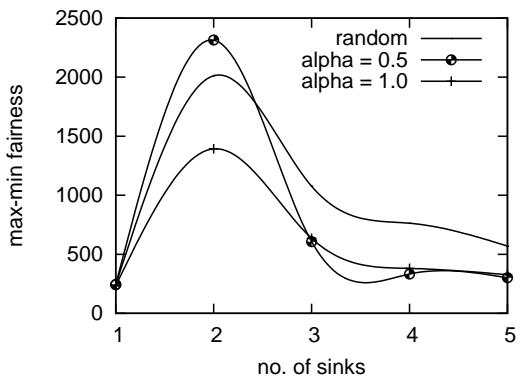

(c) Max-Min Fairness

Fig. 3. Performance of proposed heuristic in network with varying number of sinks.

TABLE II

ADMISSION CONTROL IN NETWORK WITH 32 SOURCES AND 2 SINKS

\begin{tabular}{|c||c|c||c|c||c|c||c|c|}
\hline \multicolumn{1}{|c||}{} & \multicolumn{2}{c||}{ random } & \multicolumn{2}{c||}{$\alpha=0.1$} & \multicolumn{2}{c|}{$\alpha=0.5$} & \multicolumn{2}{c|}{$\alpha=1.0$} \\
\cline { 2 - 8 }$P_{s}$ & $N_{a}$ & $N_{s}$ & $N_{a}$ & $N_{s}$ & $N_{a}$ & $N_{s}$ & $N_{a}$ & $N_{s}$ \\
\hline 0.1 & 26.4 & 1.00 & 30.7 & 1.00 & 23.1 & 1.00 & 16.6 & 1.00 \\
0.2 & 20.2 & 1.00 & 25.2 & 0.99 & 16.6 & 1.00 & 15.9 & 1.00 \\
0.3 & 16.6 & 0.99 & 24.2 & 0.98 & 16 & 1.00 & 13.6 & 1.00 \\
0.4 & 14 & 0.99 & 23.6 & 0.96 & 15.9 & 1.00 & 9.8 & 1.00 \\
0.5 & 11.5 & 1.00 & 23.1 & 0.88 & 15.5 & 1.00 & 8.7 & 1.00 \\
0.6 & 9.4 & 1.00 & 21.6 & 0.80 & 13.6 & 1.00 & 8.7 & 1.00 \\
0.7 & 7.5 & 1.00 & 19.8 & 0.73 & 11.5 & 0.97 & 8.4 & 1.00 \\
0.8 & 6 & 1.00 & 18.9 & 0.64 & 9.8 & 0.93 & 8.3 & 1.00 \\
0.9 & 4 & 1.00 & 17.5 & 0.49 & 9.6 & 0.86 & 8.1 & 0.99 \\
1 & 1 & 1.00 & 16.6 & 0.23 & 8.7 & 0.92 & 8 & 0.99 \\
\hline
\end{tabular}

number of time slots to sensors that are nearer to the sink, thus permitting a smaller number of sensors into the system. For $\alpha=0.5$, for required $P_{s} \geq 0.4$, the proposed scheme admits more nodes into the system as compared to the random case. In particular, the minimum number of admitted sensors and sensors satisfying the required $P_{s}$ is at least equal to number of time slots available, while $N_{s}$ goes to 1 for random allocation under very high load. It is interesting to note that for admission control purpose, $\alpha=0.1$ works well for low to medium load where significantly more sensors can be admitted. The tradeoff is that some of these nodes attain less than the required $P_{s}$. For example, with required $P_{s}=0.5$, twice the number of sensors can be admitted, but $12 \%$ of these nodes have $P_{s}$ less than 0.5 .

\section{CONCLUSION}

Slotted-Aloha is a viable candidate as a MAC protocol in TH-UWB networks, due to its simplicity and the impulsive, carrier-less nature of UWB transmissions. With TH-UWB, sensor nodes in the network are able to select different timehopping slots for transmissions to the sinks, thus providing some form of multiple access even at the physical layer.

In this paper, we study the time slot allocation problem in multi-sink single-hop UWB networks and prove that the optimal allocation of TH slots to the sensors is NP-hard. A greedy heuristic is then proposed to allocate $\mathrm{TH}$ slots to groups of nodes that are of similar distances to any sink together. This has the dual objectives of improving throughput by encouraging concurrent transmissions to multiple sinks, as well as achieving fairness by segregating the contention between sensors that are placed at different distances from the sinks. Simulation results show that our scheme is able to perform well, and can also be used for admission control.

As part of future work, we will study the routing problem in multi-sink multi-hop UWB-based networks and explore ways of achieving high throughput with admission control.

\section{ACKNOWLEDGMENT}

This work is done under the USCAM-CQ project which is part of the UWB-Sentient Computing Research Programme funded by SERC, A*STAR Singapore.

\section{REFERENCES}

[1] "Wireless medium access control (MAC) and physical layer (PHY) specification for low-rate wireless personal area networks (LR-WPANs)," IEEE Standards Draft 802.15.4a, 2006.

[2] D. Porcino and W. Hirt, "Ultra-wideband radio technology: potential and challenges ahead," IEEE Communications Magazine, vol. 41, no. 7, 2003.

[3] F. C. Commission, "First report and order, revision of part 15 of the commission's rules regarding ultra-wideband transmission systems," 2002.

[4] A. Das and D. Dutta, "Data acquisition in multiple-sink sensor networks," ACM SIGMOBILE Mobile Computing and Communications Review, vol. 9, no. 3, 2005 .

[5] Y. T. Hou, Y. Shi, and H. D. Sherali, "Optimal base station selection for anycast routing in wireless sensor networks," IEEE Transactions on Vehicular Technology, vol. 55, no. 3, 2006.

[6] P. Ciciriello, L. Mottola, and G. P. Picco, "Efficient routing from multiple sources to multiple sinks in wireless sensor networks," in 4th European Conference on Wireless Sensor Networks (EWSN 2007), 2007.

[7] Z. Vincze, R. Vida, and A. Vidacs, "Deploying multiple sinks in multihop wireless sensor networks," in IEEE International Conference on Pervasive Services, 2007.

[8] H. X. Tan, R. Patro, M. C. Chan, P. Y. Kong, and C. K. Tham, "Performance of Slotted-Aloha over TH-UWB," in IEEE International Conference on Ultra-Wideband (ICUWB), 2007.

[9] C. Intanagonwiwat and D. De Lucia, "The sink-based anycast routing protocol for ad hoc wireless sensor networks," Computer Science Department, University of Southern California, Tech. Rep., 1999.

[10] X. Liu and M. Haenggi, "Throughput Analysis of Fading Sensor Networks with Regular and Random Topologies," EURASIP Journal on Wireless Communications and Networking, vol. 5, no. 4, 2005.

[11] Qualnet Network Simulator, Scalable Network Technologies, Inc. [Online]. Available: www.qualnet.com 